Pontifícia Universidade Católica

DO RIO DE JANEIRO

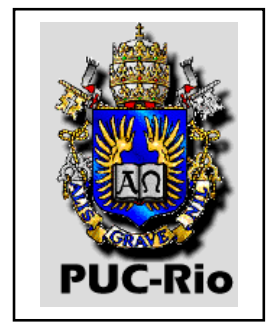

Ricardo Dias de Souza

\title{
AVALIAÇÃO ESTRUTURAL DE DUTOS COM DEFEITOS DE CORROSÃO REAIS
}

Dissertação de Mestrado

Dissertação apresentada ao Programa de Pós-graduação em Engenharia Mecânica da PUC-Rio como requisito parcial para obtenção do título de Mestre em Ciências de Engenharia Mecânica.

Orientador: José Luiz de França Freire Co-orientador: Adilson Carvalho Benjamin 
Pontifícia Universidade Católica

DO RIO DE JANEIRO

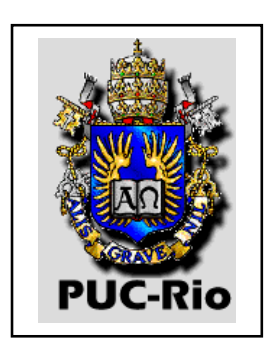

Ricardo Dias de Souza

\section{Avaliação Estrutural de Dutos com Defeitos de Corrosão Reais}

Dissertação apresentada como requisito parcial para obtenção do grau de Mestre pelo Programa de Pósgraduação em Engenharia Mecânica da PUC-Rio. Aprovada pela Comissão Examinadora abaixo assinada.

Prof. José Luiz de França Freire Orientador Departamento de Engenharia Mecânica - PUC-Rio

Eng. Adilson Carvalho Benjamin Co-orientador Petrobras/Cenpes

Prof. Arthur Martins Barbosa Braga Departamento de Engenharia Mecânica - PUC-Rio

Eng. Luiz Cláudio de Marco Meniconi Petrobras/Cenpes

Prof. Ronaldo Domingues Vieira Departamento de Engenharia Mecânica - PUC-Rio

Prof. Ney Augusto Dumont Coordenador Setorial do Centro Técnico Científico - PUC-Rio 
Todos os direitos reservados. É proibida a reprodução total ou parcial do trabalho sem autorização da universidade, do autor e do orientador.

\section{Ricardo Dias de Souza}

Graduou-se em Engenharia Civil na UFRJ (Universidade Federal do Rio de Janeiro) em 1986. Cursou Engenharia de Petróleo na Petrobras em 1987 e Engenharia de Dutos na PUC-Rio em 1999. Atuou nas áreas de completação de poços de petróleo, análise de viabilidade técnica-econômica de projetos de produção de petróleo e análise de projetos de engenharia civil. Participou de cursos e congressos, no Brasil e no exterior, na área de engenharia de dutos. É responsável pela análise de integridade estrutural de dutos na Transpetro/DT/Suporte/SE/ Conf.

Ficha Catalográfica

Souza, Ricardo Dias de

Avaliação estrutural de dutos com defeitos de corrosão reais / Ricardo Dias de Souza; orientador: José Luiz de França; co-orientador: Adilson Carvalho Benjamin. - Rio de Janeiro : PUC, Departamento de Engenharia Mecânica, 2003.

[17], 112 f. : il. ; $30 \mathrm{~cm}$

Dissertação (mestrado) - Pontifícia Universidade Católica do Rio de Janeiro, Departamento de Engenharia Mecânica.

Inclui referências bibliográficas.

1. Engenharia mecânica - Teses. 2. Dutos. 3. Defeitos de corrosão. 4. Avaliação estrutural. 5. Métodos de avaliação. I. França, José Luiz de. II. Benjamin, Adilson Carvalho. III. Pontifícia Universidade Católica do Rio de Janeiro. Departamento de Engenharia Mecânica. IV. Título.

CDD: 621 
Dedico esta tese aos meus pais Iran e Jeanette que são os principais responsáveis pela minha formação profissional e que sempre me apoiaram e me deixaram completamente à vontade para seguir os caminhos que considerei serem os melhores para mim. Considero esta tese um pequeno fruto de toda a dedicação despendida por eles durante a minha vida.

Sou eternamente grato. 


\section{Agradecimentos}

Ao meu orientador, Professor José Luiz da França Freire, pela orientação, aprendizado e atenção durante o curso de Engenharia de Dutos, a realização das cadeiras de mestrado e na elaboração da tese.

Ao meu Co-orientador Adilson Carvalho Benjamin, pelo aprendizado, orientação e oportunidade de desenvolver este trabalho ao seu lado.

À minha esposa Heloisa, pelo apoio e referência profissional que são um estímulo para a vida.

À minha filha Vívian que com seus rabiscos em meus livros e apontamentos pedindo atenção, sempre me alegrou e promoveu uma descontração agradável .

Aos meus sogros Cléa e Décio pelo suporte durante o período de tese.

Aos meus colegas de trabalho Richard Ward, José Gabriel Tinoco, Antônio Geraldo de Sousa e Minoru Matsuura, que como gerentes da Petrobras, aprovaram o meu pedido para cursar o mestrado e sempre me incentivaram e apoiaram.

Ao professor Ronaldo Domingues Vieira, pela atenção durante a realização dos ensaios e discussão dos resultados.

Ao colega de trabalho André Reyes, por conferir os resultados e tabelas e na elaboração de desenhos, tornando o trabalho confiável.

Ao Departamento de Engenharia Mecânica da PUC-Rio, em especial à Roseli Marins. 
Aos meus amigos Mario Pezzi, José Alberto Chouin, Sueli Tolmasquim pelos trabalhos realizados juntos e pelos momentos agradáveis durante o período em que cursamos as cadeiras do mestrado.

Aos professores que participaram da Comissão examinadora.

A todos que não foram citados acima, porém que tiveram participação na elaboração da minha tese. 


\section{Resumo}

Souza, Ricardo Dias de. Avaliação Estrutural de Dutos com Defeitos de Corrosão Reais. Rio de janeiro, 2003, 110p. Dissertação de Mestrado Departamento de Mecânica, Pontifícia Universidade Católica do Rio de Janeiro.

A avaliação estrutural de dutos com defeitos de corrosão vem sendo estudada desde o final da década de 60. A partir dos conceitos da Mecânica da Fratura, foram elaboradas expressões semi-empíricas que permitiram estimar a pressão de ruptura de defeitos de corrosão. Desde então, essas expressões foram sendo ajustadas e aprimoradas por testes de ensaios destrutivos e análise de elementos finitos. Os principais métodos desenvolvidos são o ASME B31G, 085dL, "Effective Area", DNV RP F-101 (defeitos isolados) e DNV RP F-101 (defeitos complexos). Esta tese foi elaborada utilizando alguns dos ensaios programados para o projeto Produt 600536, e parte dos seus resultados foi aproveitada neste projeto. Para o trabalho de tese, foram utilizados cinco espécimes tubulares de aço API 5L X46, com 3,0 m de comprimento aproximado, diâmetro nominal de 457,2 mm e espessura nominal de 6,35 mm. Estes espécimes continham defeitos reais de corrosão interna, do tipo longo, localizados na geratriz inferior, e foram retirados do oleoduto Orbel I, pertencente à Petrobras, durante a sua obra de reabilitação, em 2001. Os defeitos de corrosão foram mapeados com medições manuais por ultra-som espaçadas em $20 \mathrm{~mm}$ e com medições mecanizadas CSCAN espaçadas em 5mm. Para cada espécime, foram realizados ensaios de tração em 4 corpos de prova, sendo 2 corpos retirados transversalmente e 2 longitudinalmente. Estes espécimes foram instrumentados com extensômetros de resistência elétrica e pressurizados até a ruptura. Para cada espécime, foram levantados diversos perfis de corrosão em função do comprimento estabelecido para o defeito e do tipo de medição (manual ou mecanizada). A pressão de ruptura foi estimada pelas equações dos métodos ASME B31G, 085dL, "Effective Area", DNV RP F-101 (defeitos isolados) e DNV RP F-101 (defeitos complexos), utilizando planilha Excel e/ou os programas computacionais RSTRENG e DNV 
RP F-101. Os valores de pressão de ruptura estimados para os espécimes, utilizando os métodos acima relacionados, foram comparados com as pressões de ruptura reais, obtidas nos ensaios de pressão. Os resultados confirmaram o conservadorismo embutido no método ASME B31G e comprovaram que os métodos "Effective Area” e DNV RP-F101 (complexo), que utilizam o perfil de corrosão, apresentam resultados melhores que os métodos ASME B31G e 085dL e podem ser considerados uma boa ferramenta para avaliar defeitos de corrosão, considerando somente carregamento de pressão interna.

\section{Palavras-chave}

Dutos; defeitos de corrosão; avaliação estrutural; métodos de avaliação. 


\section{Abstract}

Souza, Ricardo Dias de. Structural Assessment of Pipelines with Real Corrosion Defects. Rio de janeiro, 2003, 110p. MSc. Dissertation Departamento de Mecânica, Pontifícia Universidade Católica do Rio de Janeiro.

Structural assessment of pipelines with corrosion defects has been studied since the late 1960s. From the principles of fracture mechanics, semi-emprical mathematical expressions have been developed for predicting burst pressure of corroded pipes. Subsequently, these expressions have been modified and calibrated based on the results from finite element analyses and laboratory burst tests. The main methods are ASME B31G, Modified B31G (version $0.85 \mathrm{dL}$ ), Effective Area and DNV RP-F101 for single and complex shaped defects. This thesis was done utilizing some laboratory tests from Produt 600536 Project. For this study, five specimens with 3.0 meters each were removed from Petrobras pipeline (Orbel I), during its rehabilitation. The pipe material was API 5L X46, 18 " diameter and 0.25 "wall thickness. These specimens had real internal corrosion with very long defect length, at the 6:00 o'clock position. The corrosion defects were mapped by manual ultrasound, at $20 \mathrm{~mm}$ intervals, and mecanized ultrasonic measurements CSCAN at $5 \mathrm{~mm}$ intervals. For each pipe, the yield strength and ultimate strength were determined by tension tests of 4 specimens, 2 removed from transverse and 2 longitudinal. Every pipe specimen was monitored by strain gages and pressurized up to the point of rupture. For each specimen different profiles were determined depending on the length defined for the defect and for the intervals of measurements. The burst pressure was predicted by the following methods: ASME B31G, Modified B31G (version 0.85 dL), Effective Area and DNV RP-F101 for single and complex defects. These predicted rupture pressures were compared with the real burst pressures. The results confirmed the conservatism of the ASME B31G method and demonstrated that the Effective Area and DNV RP-F101 for complex shaped defects methods can be considered 
good for evaluating longitudinal corrosion defects, considering only internal pressure load.

\section{Keywords:}

Pipelines; corrosion defects; structural assessment; assessment methods. 


\section{Sumário}

1- Introdução 1

1.1 - Motivação do tema de tese 3

1.2 - Intenção de tese e seu encaminhamento 5

1.3 - Apresentação dos capítulos da tese $\quad 8$

2 - Integridade estrutural de dutos com defeitos de corrosão 10

2.1 - Inspeção em dutos 10

2.1.1 - Histórico dos "pigs" instrumentados 11

2.1.2 - Especificação e exigências para a inspeção com "pig" instrumentado 11

2.1.3 - Inspeção com "pig" instrumentado de corrosão 12

2.1.4 - Mapeamento dos defeitos de corrosão 13

2.1 .5 - Periodicidade de inspeção com "pig" de corrosão 14

2.2 - Avaliação de defeitos por níveis de complexidade 16

2.3 - Métodos de análise de defeitos de corrosão 22

2.3.1 - Método ASME B31G 25

2.3.2 - Método $0.85 \mathrm{dL} \quad 27$

2.3.3 - Método "Effective Area” 28

2.3.4 - Método DNV RP-F101 30

2.3.4.1 - Avaliação para defeito isolado 32

2.3.4.2 - Avaliação para defeito de geometria complexa 33

2.3.5 - Outros cálculos realizados 36

3 - Procedimento experimental 39

3.1 - Características dos espécimes tubulares 39

3.2 - Mapeamento manual de espessura de parede por ultra-som 39

3.3 - Mapeamento mecanizado da espessura de parede do tipo CSCAN 41

3.4 - Ensaios de tração do material dos espécimes 43

3.5 - Confecção dos espécimes tubulares 44 
4 - Determinação das pressões de ruptura 51

4.1 - Espessura de parede 51

4.2 - Diâmetro 51

4.3 - Resistência ao escoamento e à tração 52

4.4 - Perfil de Corrosão 52

4.5 - Cálculo da pressão de ruptura 60

5 - Análise dos resultados 69

5.1 - Análise quanto à resistência ao escoamento e à tração dos materiais 69

5.2 - Análise do volume injetado e da deformação plástica dos espécimes 70

5.3 - Análise das parcelas que compõem as equações que estimam a pressão de ruptura $\quad 71$

5.4 - Análise dos resultados das pressões de ruptura estimadas $\quad 72$

5.4.1 - Análise individual por espécime 73

5.4.2 - Análise geral quanto aos métodos de cálculo 76

5.4.3 - Análise geral quanto aos perfis de corrosão 78

5.5 - Análise comparativa utilizando $t$ e $D$ nominais por $t$ e $D$ medidos 79

5.6 - Análise adicionais com o espécime T02 81

5.7 - Comparação entre perfil River Bottom e Perfil Geratriz de Ruptura 82

6 - Conclusões e sugestões para trabalhos futuros 83

7 - Referências bibliográficas 90

Apêndice A - Gráfico taxa de corrosão x probabilidade acumulativa 94 Apêndice B - Telas dos programas Rstreng e DNVRP-F101 95 
Apêndice C - Mapeamento Manual e CSCAN dos Espécimes T04, T05, T06 e T10

Apêndice D - Valores de Prup e Prup', perfis river bottom e geratriz de ruptura 106

Apêndice $\mathrm{E}$ - Análise das pressões de ruptura 


\section{Lista de Figuras}

Figura 1 - Avaliação de defeitos por níveis de complexidade 21

Figura 2 - Área longitudinal do material perdido 23

Figura 3 - Representação da área longitudinal perdida por meio de uma área parabólica e retangular

Figura 4 - Detalhe dos comprimentos para o cálculo pelo método "Effective Area"

Figura 5 - Definição da área de patch $\left(A_{\text {patch }}\right)$ e de pit $\left(A_{\text {pit }}\right)$

Figura 6 - Exemplo de agrupamento de defeitos adjacentes para interação

Figura 7 - Medição manual por ultra-som 40

Figura 8 - Medição mecanizada por ultra-som $\quad 42$

Figura 9 - Espécime T04 instrumentado e sendo pressurizado $\quad 47$

Figura 10 - Espécime T04 no instante da ruptura 48

Figura 11 - Espécime T04 após a ruptura 48

Figura 12 - Gráfico Volume Injetado x Pressão espécime T02 49

Figura 13 - Mapeamento manual por ultra-som do espécime T02 com L=1980 mm

Figura 14 - Mapeamento manual por ultra-som do espécime T02 com L $=980 \mathrm{~mm}$

Figura 15 - Mapeamento mecanizado CSCAN ímpar por ultra-som do espécime $\mathrm{T} 02$ com L $=990 \mathrm{~mm}$

Figura 16 - Mapeamento mecanizado CSCAN par por ultra-som do espécime T02 com L=990 mm

Figura 17 - Gráfico de $P_{\text {rup }}$ X método de cálculo do Espécime T 0264

Figura 18 - Gráfico de $P_{\text {rup }} \times$ método de cálculo do Espécime T 0464

Figura 19 - Gráfico de $P_{\text {rup }} \times$ método de cálculo do Espécime T $05 \quad 65$

Figura 20 - Gráfico de $P_{\text {rup }} \times$ método de cálculo do Espécime T $06 \quad 65$

Figura 21 - Gráfico de $P_{\text {rup }} \times$ método de cálculo do Espécime T 10 
Figura 22 - Gráfico de $P_{\text {rup' }}$ x método de cálculo do Espécime T 02 Todos perfis

Figura 23 - Gráfico de $\mathrm{P}_{\text {rup }}$ X método de cálculo do Espécime T 02 Medição manual

Figura 24 - Gráfico de $\mathrm{P}_{\text {rup }}$ ' x método de cálculo - Espécime T 02 -

CSCAN - L $500 \mathrm{~mm}$

Figura 25 - Gráfico de $\mathrm{P}_{\text {rup }}$ x método de cálculo-Espécime T 02-

CSCAN - L $1000 \mathrm{~mm}$ 68

Figura 26 - Gráfico taxa de corrosão x probabilidade acumulativa 94

Figura 27 - Tela do programa Rstreng 95

Figura 28 - Tela do programa DNVRP-F101 para defeitos isolados 96 Figura 29 - Tela do programa DNVRP-F101 para defeitos de geometria complexa

Figura 30 - Mapeamento manual por ultra-som - Espécime T04 $\mathrm{L}=1980 \mathrm{~mm}$ 98

Figura 31 - Mapeamento CSCAN ímpar - Espécime T04 - L=990 $\mathrm{mm}$ 99

Figura 32 - Mapeamento CSCAN par - Espécime T04 - L=990 mm 99 Figura 33 - Mapeamento manual por ultra-som - Espécime T05 $\mathrm{L}=1980 \mathrm{~mm}$ 100

Figura 34 - Mapeamento CSCAN ímpar - Espécime T05 - L=990 $\mathrm{mm}$

Figura 35 - Mapeamento CSCAN par - Espécime T05 - L=990 mm

Figura 36 - Mapeamento manual por ultra-som - Espécime T06 $\mathrm{L}=1980 \mathrm{~mm}$

Figura 37 - Mapeamento CSCAN ímpar - Espécime T06 $\mathrm{L}=990 \mathrm{~mm}$ 103

Figura 38 - Mapeamento CSCAN par - Espécime T06 - L=990 mm 103 Figura 39 - Mapeamento manual por ultra-som - Espécime T10 $\mathrm{L}=1980 \mathrm{~mm}$

Figura 40 - Mapeamento CSCAN ímpar - Espécime T10 $\mathrm{L}=990 \mathrm{~mm}$

Figura 41 - Mapeamento CSCAN par - Espécime T10 - L=990 mm 


\section{Lista de tabelas}

Tabela 1 - Resumo das equações dos métodos estudados 38

Tabela 2 - Valores dos ensaios de tração 44

Tabela 3 - Localização dos extensômetros em cada espécime 46

Tabela 4 - Pressão de ruptura e volume injetado 48

Tabela 5 - Pressão e volume injetado no início da deformação plástica 49

Tabela 6 - Valores de espessura na região compreendida entre 8 , 12 e $4 \mathrm{~h}$

Tabela 7 - Diâmetro dos espécimes 52

Tabela 8 - Valores médios das resistências ao escoamento e à tração 52

Tabela 9 - Comprimento dos espécimes sem tampo 53

Tabela 10 - Perfis com espessuras e diâmetros medidos 56

Tabela 11 - Perfis com espessuras e diâmetros nominais 56

Tabela 12 - Pressão de ruptura estimada para cada método de cálculo, pressão de ruptura real e pressão de ruptura estimada para tubo novo, em kgf/ $/ \mathrm{cm}^{2} \quad 62$

Tabela 13 - Valores de $\mathrm{P}_{\text {rup }}$, para cada método de cálculo 63

Tabela 14 - Razão entre valores de resistência ao escoamento e à tração 70

Tabela 15 - Volume dos espécimes e volume injetado até a ruptura 70

Tabela 16 - Razão entre os $\sigma_{\text {flow }}$ 's dos métodos estudados $\quad 71$

Tabela 17 - Variação e média da $P_{\text {rup }}$ para cada método de cálculo 76

Tabela 18 - Razão pressão estimada de ruptura com valores de t e D nominais por $t$ e $D$ medidos, Métodos ASME B31G, 0,85 dL e "Effective Area".

Tabela 19 - Razão pressão estimada de ruptura com valores de t e D nominais por t e D medidos, Métodos DNV RP-F101(complexo) e DNV RP-F101(isolado) 
Tabela $20-P_{\text {rup }}\left(\mathrm{kgf} / \mathrm{cm}^{2}\right)$ para Espécime T02, perfis river bottom e geratriz de ruptura

Tabela $21-P_{\text {rup }}\left(\mathrm{kgf} / \mathrm{cm}^{2}\right)$ para Espécime T04, perfis river bottom e geratriz de ruptura

Tabela $22-P_{\text {rup }}\left(\mathrm{kgf} / \mathrm{cm}^{2}\right)$ para Espécime T05, perfis river bottom e geratriz de ruptura

Tabela 23- $P_{\text {rup }}\left(\mathrm{kgf} / \mathrm{cm}^{2}\right)$ para Espécime T06, perfis river bottom e geratriz de ruptura

Tabela $24-P_{\text {rup }}\left(\mathrm{kgf} / \mathrm{cm}^{2}\right)$ para Espécime T10, perfis river bottom e geratriz de ruptura

Tabela 25- $\mathrm{P}_{\text {rup }}$, para Espécime T02, perfis river bottom e geratriz de ruptura

Tabela 26- $\mathrm{P}_{\text {rup }}$, para Espécime T04, perfis river bottom e geratriz de ruptura

Tabela 27- $\mathrm{P}_{\text {rup }}$ para Espécime T05, perfis river bottom e geratriz de ruptura

Tabela 28- $\mathrm{P}_{\text {rup }}$, para Espécime T06, perfis river bottom e geratriz de ruptura

Tabela 29- $\mathrm{P}_{\text {rup }}$, para Espécime T10, perfis river bottom e geratriz de ruptura 